\title{
Role of Maintenance Gemcitabine in Advanced Carcinoma Gallbladder
}

\author{
Manish Sharma ${ }^{1}$ Vineet Talwar ${ }^{1} \quad$ Udip Maheshwari ${ }^{1}$ Venkata Pradeep Babu Koyyala ${ }^{1}$ \\ Varun Goel ${ }^{1}$ Sumit Goyal ${ }^{1}$ Prasanta Kumar Dash ${ }^{1} \quad$ Ullas Batra ${ }^{1} \quad$ Rajat Bajaj $^{2}$ Abhishek Yadav ${ }^{3}$ \\ Pankaj Goyal ${ }^{1}$ Dinesh Chandra Doval ${ }^{1}$
}

${ }^{1}$ Department of Medical Oncology, Rajiv Gandhi Cancer Institute and Research Center, New Delhi, India

${ }^{2}$ Department of Medical Oncology, Fortis Escorts Hospital, Faridabad, Haryana, India

${ }^{3}$ Department of Medical Oncology, Fortis Escorts Hospitals, Delhi, India

\begin{abstract}
Address for correspondence Udip Maheshwari, MD, DNB medical oncology, Department of Medical Oncology, Rajiv Gandhi Cancer Institute and Research Center, New Delhi, India (e-mail: udip.the.drift.king@gmail.com).
\end{abstract}

\section{South Asian J Cancer 2021;9:204-208.}

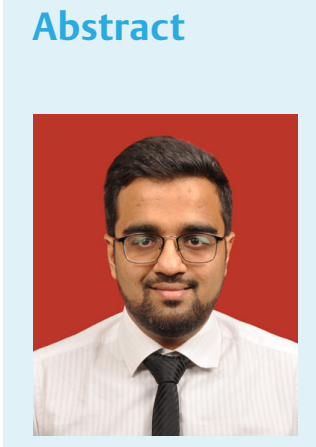

Udip Maheshwari, MD

Keywords

- gallbladder cancer

- gemcitabine

- maintenance

- progression-free survival
Objective The aim of this study is to investigate the effects of gemcitabine maintenance on progression-free survival (PFS) in patients with metastatic gallbladder cancer (GBC).

Materials and Methods Sixty patients with unresectable or metastatic GBC having ongoing response to treatment with initial six cycles of gemcitabine and a platinum-based doublet chemotherapy were prospectively randomized on day 21 of the 6th cycle in 1:1 fashion to receive either maintenance gemcitabine $1 \mathrm{~g} / \mathrm{m}^{2}$ intravenously on day 1 and day 8 of three weekly cycle or observation. Survival analysis was performed using the Kaplan-Meier method and comparisons by the logrank test. A $p$-value $<0.05$ was considered as statistically significant.

Results Of 60 patients, a total of 56 were available for final analysis. The median PFS was 4.7 months (3.1-6.3) in gemcitabine arm and 2.6 months (2.4-2.8) in observation arm, hazard ratio (HR) 0.196 (95\% confidence interval [Cl]: 0.1-0.39), $p<0.001$. Median overall survival in gemcitabine arm was 12.4 months $(9.15-15.6)$ as opposed to 9.9 months (8.29-11.5) in observation arm, HR 0.76 (95\% Cl: $0.43-1.35), p=0.354$. The grade 3 or 4 side effects in maintenance arm were transaminitis (17.9\%), thrombocytopenia (17.8\%), neutropenia (14.2\%), and febrile neutropenia $(7.1 \%)$.

Conclusions Maintenance gemcitabine therapy in unresectable/metastatic GBC patients responding to first-line gemcitabine and platinum treatment contributes to increase PFS with minimal and manageable side effects.

\section{Introduction}

Gallbladder cancer (GBC) is a significant health problem. Very high incidence has been reported in women in countries such as Chile, Poland, India, Israel, Pakistan, Ecuador, South Korea, and Japan, whereas it is a rare cancer in most Western countries and the United States. ${ }^{1-3}$ In India, the regions with the highest incidence of $\mathrm{GBC}$ are Northern
How to cite this article : Sharma M, Talwar V, Maheshwari U, Babu Koyyala, Goyal V, Dash P.K, Batra U, Bajaj R, Yadav A, Goyal P, Doval D. C. Role of Maintenance Gemcitabine in Advanced Carcinoma Gallbladder South Asian J Cancer 2021;9(4):204-208. and Northeastern states of Uttar Pradesh, Bihar, Orissa, West Bengal, and Assam. ${ }^{4}$ Majority of these patients present late in the advanced, surgically unresectable stage and only palliative treatment and/or supportive care can be offered to them. Median survival for patients presenting with the unresectable disease is 6 months, with fewer than $5 \%$ of patients surviving 1 year. ${ }^{5}$ The majority of studies on the treatment

(C) 2021. MedIntel Services Pvt Ltd.

This is an open access article published by Thieme under the terms of the Creative Commons Attribution-NonDerivative-NonCommercial-License, permitting copying and reproduction so long as the original work is given appropriate credit. Contents may not be used for commercial purposes, or adapted, remixed, transformed or built upon. (https://creativecommons.org/licenses/by-nc-nd/4.0/)

Thieme Medical and Scientific Publishers Pvt. Ltd., A-12, 2nd Floor, Sector 2, Noida-201301 UP, India 
of metastatic disease are heterogeneous and have included patients from all subsites of biliary tract cancers, that is, gallbladder carcinoma, cholangiocarcinoma, and periampullary cancers. Phase III randomized studies addressing the treatment of metastatic disease are very few.

The current standard of care for stage 4 GBC comes from a randomized NCRN (National Cancer Research Network) phase III $\mathrm{ABC}-02^{6}$ study that established a gemcitabine and cisplatin combination as first-line therapy. In this study, overall survival (OS) was significantly greater with gemcitabine and cisplatin combination therapy versus gemcitabine alone (11.7 vs. 8.1 months), as was median progression-free survival (PFS) (8 vs. 5 months). In another large phase III randomized control study from India, Sharma et $\mathrm{al}^{7}$ randomized 81 patients with unresectable or metastatic GBC reported median OS of 4.5, 4.6, and 9.5 months for the patients randomized to best-supportive care, Bolus 5 FU/Folinic acid regimen, and the MGEMOX arms ( $p=0.039$ ), respectively. PFS was reported as $2.8,3.5$, and 8.5 months for the three groups ( $p<0.001$ ), respectively. In both of these trials, the chemotherapy was well tolerated. Hence, at best, the maximum median PFS achievable is 8 to 8.5 months. Hence, low survival rates obtained with these agents and unavoidable disease progression occurring after discontinuing the firstline chemotherapy mandate further research to find more effective and tolerable treatments to delay disease progression and improve survival in advanced GBC.

At present, there is an increasing interest to investigate the role of maintenance in various advanced solid tumors. Few examples of solid cancers where maintenance strategy has worked are advanced/metastatic nonsmall cell lung cancer, metastatic colorectal cancer, and ovarian cancer. Using the platinum-based chemotherapy could be one option for increasing survival, but bone marrow suppression, ototoxicity, and nephrotoxicity are its limitations. Hence, in the present study, we evaluated the maintenance treatment with gemcitabine in patients with advanced GBC after standard chemotherapy.

\section{Materials and Methods}

\section{Study Design}

The study was conducted at Rajiv Gandhi Cancer Institute and Research Centre, a tertiary care cancer center located in New Delhi, India. This prospective randomized observational study was conducted in compliance with the ethical principles, and it was approved by the Independent Ethics Committee of our hospital, and all necessary regulatory approvals were obtained. The study was hospital funded and all the required compensations were as per the hospital protocol. All patients were required to sign written informed consent before randomization. We hypothesized that maintenance treatment with gemcitabine contributes to survival advantage for patients with GBC and it is a safe treatment option.

\section{Patients}

Patients were recruited between June 2015 and July 2017. All eligible patients were 18 years of age or older and had a confirmed histopathological or cytologic diagnosis of unresectable, recurrent, or metastatic GBC; the Eastern Cooperative Oncology Group performance status (PS) of $0-2$; ongoing response on initial treatment with six cycles of gemcitabine $1 \mathrm{~g} / \mathrm{m}^{2}$ day 1 and day 8 , plus either cisplatin $75 \mathrm{mg} / \mathrm{m}^{2}$ day 1 or carboplatin area under the curve 5 day 1 every three weekly regimen as assessed by response evaluation criteria in solid tumors (RECIST) criteria 1.1. All eligible patients had adequate hepatorenal and bone marrow reserves. Patients having active infection, pregnancy and lactation, uncontrollable diabetes or hypertension, other primary malignancy, and documented brain metastases were excluded from the study.

\section{Treatment}

At the end of initial six cycles of gemcitabine and platinum (either cisplatin or carboplatin)-based therapy (day 21 of cycle 6), patients were randomized into Arm A (intervention arm) and Arm B (comparator arm) using computerized block randomization. All patients underwent pretreatment evaluation. Arm A was given following chemotherapy regimen: injection gemcitabine $1 \mathrm{~g} / \mathrm{m}^{2}$ intravenously on day 1 and day 8 of three weekly cycle on outpatient basis till disease progression (either on imaging or clinical progression) or unacceptable toxicity or patient's choice, whereas arm B was kept under surveillance.

\section{Assessments}

Evaluations before each cycle of therapy included a complete history, physical examination, and assessment for toxic effects of the drugs, complete hemogram, liver function, and kidney function test. RECIST response criteria (version 1.1) were used to define the antitumor effects; responses were assessed only before randomization, after every three cycles (just prior to subsequent cycle) by clinical assessment and computed tomography/positron-emission tomography scans in arm A and every 9 weeks in arm B. The tumor response was measured as complete response (CR), partial response (PR), or stable disease (SD).

\section{Statistical Analysis}

Cohen's effect size was used to calculate the sample size. To detect effect size of 0.75 , the minimum required sample size with $80 \%$ power of the study and two-sided $\alpha$ of $5 \%$ was 28 patients per arm. Sample size was taken as 66 (33 per group) to allow for the random dropouts. At the end of initial six cycles of gemcitabine and platinum (either cisplatin or carboplatin)-based therapy (day 21 of cycle 6), patients were randomized into arm A and arm B using computerized block randomization. The final analysis was performed as per protocol basis. PFS and OS were assessed as per standard definitions. Toxic effects were categorized according to the National Cancer Institute's Common Toxicity Criteria for Adverse Events, version 4.0. Descriptive summaries were presented as a mean \pm standard deviation continuous variables and as frequencies with percentages for categorical variables. Two-sample proportion Z-test was performed using MedCalc for Windows, version 15.0 (MedCalc Software, Ostend, 
Belgium). Survival analysis was performed using KaplanMeier method and comparison between cases and control was made by log-rank test. Hazard ratio (HR) was calculated by Cox regression analysis. The data were analyzed using IBM SPSS Statistics for Windows, Version 25.0. IBM Corp, Armonk, NY, United States. A $p$-value $<0.05$ was considered statistically significant.

\section{Results}

A total of 66 patients were selected between June 2015 and July 2017 for this study of which six patients were excluded as per eligibility criteria. In the remaining 60 patients, 30 patients received maintenance gemcitabine (arm A), and 30 patients were kept under surveillance (arm B). After excluding the patients who were lost to follow-up and withdrew consent, the final analysis was performed 12 months after the last patient was recruited. At the time of the final analysis, all the 56 patients had tumor progression and hence, all 56 patients were eligible for survival analysis. Majority of patients had adenocarcinoma (92.6\%), whereas three patients had adenosquamous and one patient had squamous histology.

\section{Treatment Compliance}

Of 60 patients who were randomized between arm $\mathrm{A}$ and arm $B$, one patient withdrew consent for treatment after one cycle and one patient was lost to follow-up, whereas two patients were lost to follow-up from arm B. The median time taken to administer initial six cycles of gemcitabine and platinum-based chemotherapy was 4.5 months in arm A and 4.45 months in arm B. The median time from the randomization to the first maintenance dose was 3 days (range, 2-8 days), with the majority of patients (95\%) initiating maintenance therapy within 7 days. Total numbers of cycles of gemcitabine maintenance administered were 193. The median numbers of cycles received were 6 (range, 2-15). Total of nine cycles were delayed due to various toxicities. Five $(17.8 \%)$ patients received $<4$ cycles, 10 (35.7\%) patients received $4-6$ cycles, and 13 (46.4\%) patients received $>6$ cycles of gemcitabine maintenance. Total of $78.6 \%$ and $67.9 \%$ (10.7\%, $95 \%$ of confidence interval $[\mathrm{CI}]: 12.3-32.3, p=0.372$ ) in the maintenance and observation group received second-line FOLFOX-based chemotherapy and $21.4 \%$ and $14.2 \%$ (7.2\%, 95\% CI: $13.3-27.2, p=0.485)$ received third-line palliative chemotherapy.

\section{Survival and Disease Progression}

The final analysis was done at 12 months after the last patient enrolment when all the patients in arm A as well as arm B had progressive disease. The median PFS was 4.7 months (3.1-6.3) in arm A and 2.6 months (2.4-2.8) in arm B $(p<0.0001)$ with HR for arm A with arm B being reference was 0.196 (95\% CI: 0.1-0.39). Across all the subgroups, the median PFS was statistically significant except for those patients who had SD at the time of randomization (3.8 vs. 2.05 months, $p=0.425$ ). On subgroup analysis of arm A, it was observed that median PFS was better in patients younger than 50 years of age, locally advanced group as compared with upfront metastatic disease and those patients who had CR or PR as compared with SD before randomization $(p=0.04)$.

Median OS in arm A was 12.4 months (9.15-15.6) as opposed to 9.9 months (8.29-11.5) in arm B, $p=0.354$. Two-year survival rate was $17.9 \%$ and $15.2 \%$ in arm $A$ and arm $B$, respectively. HR for arm A with arm B as reference was 0.76 (95\% CI: 0.43-1.35). Kaplan-Meier curves are represented in -Figs. 1 and 2.

\section{Toxicity Analysis}

To detect any adverse effect of gemcitabine maintenance therapy, toxicity data analysis was done from June 2015 to July 2017 in the arm A. The adverse events were documented according to Common Terminology Criteria for Adverse Events Version 4 and were managed accordingly. A total of nine chemotherapeutic cycles were delayed due to any of the toxicity. The most common grade 3 and 4 toxicities in our study in arm A were hematological followed by liver

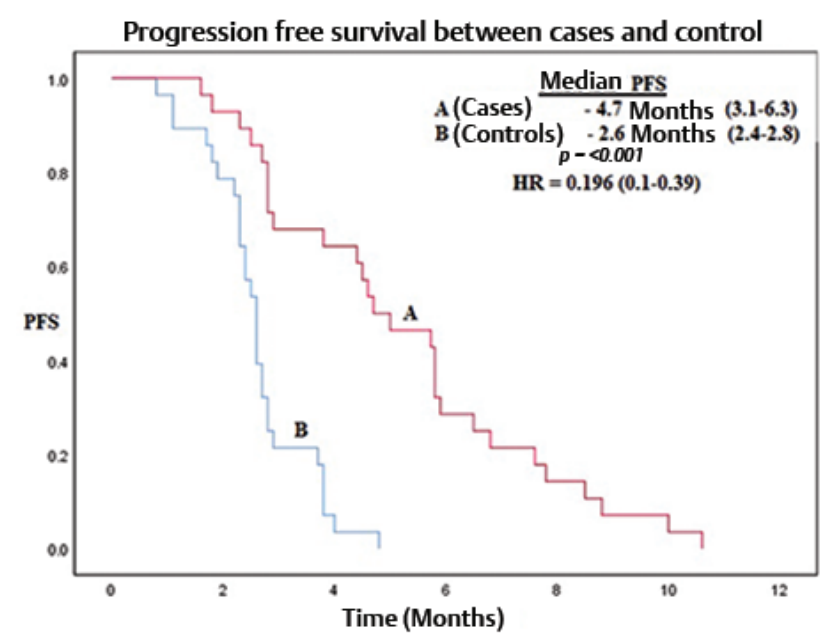

Fig. 1 Kaplan-Meier curves for progression-free survival (PFS) between arm A and arm B. HR, hazard ratio.

Overall servival between maintenance vs no maintenanace

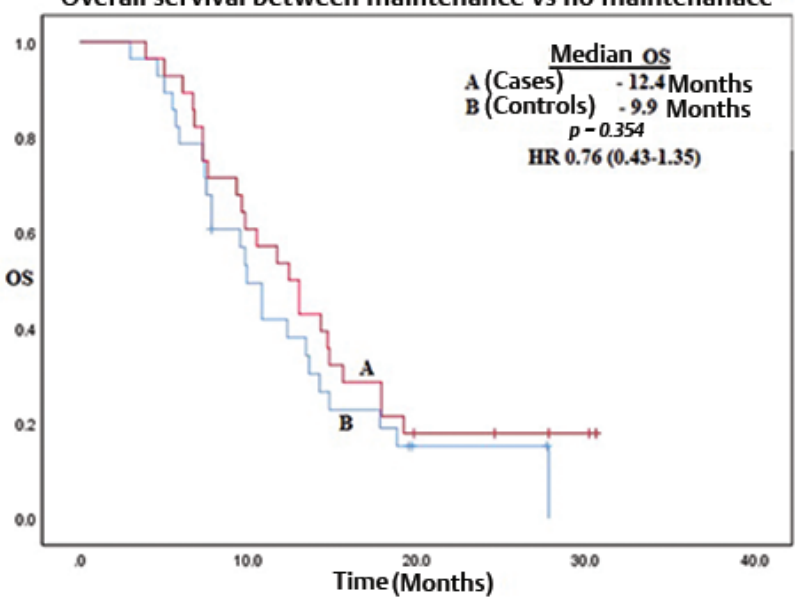

Fig. 2 Kaplan-Meier curves for overall survival (OS) between arm A and arm B. HR, hazard ratio. 
dysfunction and fatigue. None of the patients in treatment arm developed grade 4 liver dysfunction. None of our patient developed hemolytic uremic syndrome.

\section{Discussion}

GBC is an aggressive malignancy and survival in the advanced stage is less than a year. Maintenance therapy may have a role in these patients. Maintenance treatment has been successfully used in many different cancer types such as breast, lung, prostate, and ovarian cancers. ${ }^{8-14}$ To the best of our knowledge, there was no randomized study in the literature that studied the advantage of maintenance gemcitabine in patients of advanced GBC.

The majority of studies on the treatment of metastatic GBC have included heterogeneous patient population and included patients with tumors from all subsites of biliary tract, that is, gallbladder, biliary tracts, and periampullary region. The study had included patients with only GBC.

Some of the studies on GBC, where single-agent chemotherapy was used instead before the doublet chemotherapy, was established as standard therapy. In one of such earlier trials by Gallardo et al, ${ }^{15}$ in which metastatic GBC patients received single-agent gemcitabine till progression, the median survival time was 7.5 months. Another study by Penz et $\mathrm{al}^{16}$ in which single-agent gemcitabine was given till progression reported similar outcomes with a PFS of 5.6 months. However, in the present study where patients received six cycles of doublet chemotherapy as per standard protocol and the patients who responded to chemotherapy received the maintenance post randomization had a total median PFS of 9.35 months and median OS of 16.9 months (from start of induction chemotherapy to progression on maintenance). These results are certainly better than two studies that used single-agent gemcitabine till progression without initial induction therapy. This signifies the need of an induction with the doublet agent that first achieves a tumor response followed by the prolonged maintenance by single-agent gemcitabine.

In one of the earlier study, in 2004, by Doval et $\mathrm{al}^{17}$ that exclusively included advanced GBC population (having similar epidemiological profile as ours) treated with gemcitabine and cisplatin combination for six cycles reported median OS as 4.7 months. In the present study, the total median PFS and OS (from start of induction chemotherapy to progression on observation) in observation arm was 7 and 14.3 months. The probable reason seems to lie in the dramatic changes in supportive care including palliative biliary tract stenting over years that allows us to maintain dose density of the regimen and also maintains the PS of patients for the second- and third-line chemotherapies. One of the systematic reviews published in 2005 that included 13 studies used gemcitabine alone or in combination with other agents. ${ }^{18}$ Three of these studies that used cisplatin-gemcitabine regimen reported median survivals of $4.6,6.5$, and 10.4 months. The study by Valle et $\mathrm{al}^{6}$ that did not offer maintenance treatment reported median PFS and OS of 8 and 11.7 months, respectively, with cisplatin plus gemcitabine combination alone. In the present study, the total median PFS and median OS were 9.35 and 16.9 months in the maintenance arm that is certainly better than the above study implying the need for maintenance in this population. Our data provides the evidence that maintenance chemotherapy with gemcitabine is an effective treatment option for locally advanced or metastatic GBC. Patients treated with maintenance gemcitabine lived without evidence of progressive disease for 2.1 months longer than those kept under surveillance. This benefit was achieved with the use of an outpatient schedule and manageable toxicity. Across all the subgroups, that is, males, females, age $<50$, age $>50, \mathrm{CR}+\mathrm{PR}$ (before randomization), metastatic and locally advanced the PFS of the maintenance gemcitabine arm was better and the difference was statistically significant compared with the observational arm. In group of patients who had SD at the time of randomization PFS was better when given gemcitabine maintenance as compared with arm with no maintenance gemcitabine, although this was not statistically significant. Hence, the patients who have SD after initial six cycles of gemcitabine and platinum-based chemotherapy seem to be having less benefit with maintenance therapy, but this needs to be confirmed in larger studies. The tumor size and the nodal status are well established prognostic factors according to staging by AJCC (American Joint Committee on Cancer). ${ }^{19}$ In accordance with this fact, PFS in the maintenance gemcitabine arm was better in locally advanced group as compared with those patients who had upfront metastatic disease. In another study by Kayahara et $a{ }^{20}{ }^{20}$ the reported survival consistently declines with the increasing tumor size and nodal involvement and our findings correlate well with these studies. The median PFS in the present study was better and statistically significant in patients $<50$ years age as compared with those with $>50$ years age in the maintenance gemcitabine arm. This was consistent with the findings of the above-mentioned study in which patients with increasing age had poorer prognosis as compared with young patients. The reasons proposed were good PS, ability to tolerate chemotherapy, and lesser comorbidities..$^{20}$ When the population was analyzed according to the response achieved in terms of $\mathrm{CR}, \mathrm{PR}$, or SD before randomization in maintenance arm, the median PFS in CR + PR group was 5.9 months, while it was 3.8 months in patients who were having SD at the time of randomization. The difference was statistically significant $(p$ $=0.04$ ). The probable reason for the best PFS advantage in this group is that this was the group that was already responding to chemotherapy and hence continuing maintenance gemcitabine is most logical in this group. Hence, the best subset according to the present study, patients who benefit the most with prolonged gemcitabine maintenance are patients with age $<50$ years with locally advanced GBC, those in CR or PR after initial six cycles of gemcitabine and platinum-based chemotherapy prior to the start of maintenance. Although the second-line chemotherapy is not well established after progressive disease as an institutional protocol, we offered FOLFOX-based chemotherapy to these patients. Total of 78.6 and $67.9 \%$ in the maintenance and observation group received second-line FOLFOX-based chemotherapy and 21.4 and $14.2 \%$ received third-line chemotherapy. The difference 
between the two groups for next-line chemotherapies was not statistically significant, although, numerically more number of patients could be started on second- and thirdline chemotherapy. OS in the present study was not significantly different in the two groups as most patients received second- and third-line chemotherapy. Toxicity as reported by other studies was manageable and the most common toxicity in the present study in arm A was hematological followed by liver dysfunction. Overall, gemcitabine maintenance chemotherapy regimen was very well tolerated in the present study. The study has certain limitations first, that it is a observational and not an interventional study, second, it is a single institution study and for generalization of results larger randomized studies would be required, and finally, subset analysis performed in this study is unplanned and hence the conclusions need further confirmation. To best of our knowledge, this is the first prospective study to observe the role of maintenance chemotherapy in advanced and metastatic GBC; however, further randomized phase III clinical trials are required to confirm the results.

\section{Conclusions}

Maintenance chemotherapy in advanced and metastatic GBC is an effective and safe alternative especially in patients $<50$ years of age or who have a locally advanced disease at presentation or who have a PR or CR after induction chemotherapy. However, no OS advantage was observed with maintenance therapy in GBC and larger randomized phase III studies would be required to further confirm these results.

\section{Funding}

Nil.

Conflicts of Interest

There are no conflicts of interest.

\section{References}

1 Randi G, Franceschi S, La Vecchia C. Gallbladder cancer worldwide: geographical distribution and risk factors. Int J Cancer 2006;118(7):1591-1602

2 Medina E, Kaempffer AM. [Cancer mortality in Chile: epidemiological considerations]. Rev Med Chil 2001;129(10):1195-1202

3 Lazcano-Ponce EC, Miquel JF, Muñoz N, et al. Epidemiology and molecular pathology of gallbladder cancer. CA Cancer J Clin 2001;51(6):349-364

4 Nandakumar A, Gupta PC, Gangadharan P, Visweswara RN, Parkin DM. Geographic pathology revisited: development of an atlas of cancer in India. Int J Cancer 2005;116(5):740-754

5 Levy AD, Murakata LA, Rohrmann CA Jr. Gallbladder carcinoma: radiologic-pathologic correlation. Radiographics 2001;21(2):295-314, 549-555
6 Valle J, Wasan H, Palmer DH, et al; ABC-02 Trial Investigators. Cisplatin plus gemcitabine versus gemcitabine for biliary tract cancer. N Engl J Med 2010;362(14):1273-1281

7 Sharma A, Dwary AD, Mohanti BK, et al. Best supportive care compared with chemotherapy for unresectable gall bladder cancer: a randomized controlled study. J Clin Oncol 2010; 28(30):4581-4586

8 Kanda S, Goto K, Shiraishi H, et al. Safety and efficacy of nivolumab and standard chemotherapy drug combination in patients with advanced non-small-cell lung cancer: a four arms phase Ib study. Ann Oncol 2016;27(12):2242-2250

9 Zwitter M, Rajer M, Stanic K, et al. Intercalated chemotherapy and erlotinib for non-small cell lung cancer (NSCLC) with activating epidermal growth factor receptor (EGFR) mutations. Cancer Biol Ther 2016;17(8):833-839

10 Cathomas R, Crabb SJ, Mark M, et al; Swiss Group for Clinical Cancer Research SAKK. Orteronel switch maintenance therapy in metastatic castration resistant prostate cancer after first-line docetaxel: A multicenter, randomized, double-blind, placebo-controlled trial (SAKK 08/11) Prostate 2016; 76(16):1519-1527

11 Mirza MR, Monk BJ, Herrstedt J, et al; ENGOT-OV16/ NOVA Investigators. Niraparib maintenance therapy in platinum-sensitive, recurrent ovarian cancer.NEnglJMed 2016; 375(22):2154-2164

12 Ferrero JM, Hardy-Bessard AC, Capitain O, et al. Weekly paclitaxel, capecitabine, and bevacizumab with maintenance capecitabine and bevacizumab as first-line therapy for triple-negative, metastatic, or locally advanced breast cancer: Results from the GINECO A-TaXel phase 2 study. Cancer 2016;122(20):3119-3126

13 Rossi S, Schinzari G, Basso M, et al. Maintenance hormonal and chemotherapy treatment in metastatic breast cancer: a systematic review. Future Oncol 2016;12(10):1299-1307

14 Kulkarni S, Vella ET, Coakley N, et al. The use of systemic treatment in the maintenance of patients with non-small cell lung cancer: a systematic review. J Thorac Oncol 2016; 11(7):989-1002

15 Gallardo JO, Rubio B, Fodor M, et al. A phase II study of gemcitabine in gallbladder carcinoma. Ann Oncol 2001; 12(10):1403-1406

16 Penz M, Kornek GV, Raderer M, et al. Phase II trial of two-weekly gemcitabine in patients with advanced biliary tract cancer. Ann Oncol 2001;12(2):183-186

17 Doval DC, Sekhon JS, Gupta SK, et al. A phase II study of gemcitabine and cisplatin in chemotherapy-naive, unresectable gall bladder cancer. Br J Cancer 2004;90(8):1516-1520

18 Dingle BH, Rumble RB, Brouwers MC; Cancer Care Ontario's Program in Evidence-Based Care's Gastrointestinal Cancer Disease Site Group. The role of gemcitabine in the treatment of cholangiocarcinoma and gallbladder cancer: a systematic review. Can J Gastroenterol 2005;19(12):711-716

19 Wakabayashi $\mathrm{H}$, Ishimura $\mathrm{K}$, Hashimoto $\mathrm{N}$, Otani $\mathrm{T}$, Kondo A, Maeta $\mathrm{H}$. Analysis of prognostic factors after surgery for stage III and IV gallbladder cancer. Eur J Surg Oncol 2004;30(8):842-846

20 Kayahara M, Nagakawa T, Nakagawara H, Kitagawa H, Ohta T. Prognostic factors for gallbladder cancer in Japan. Ann Surg 2008;248(5):807-814 